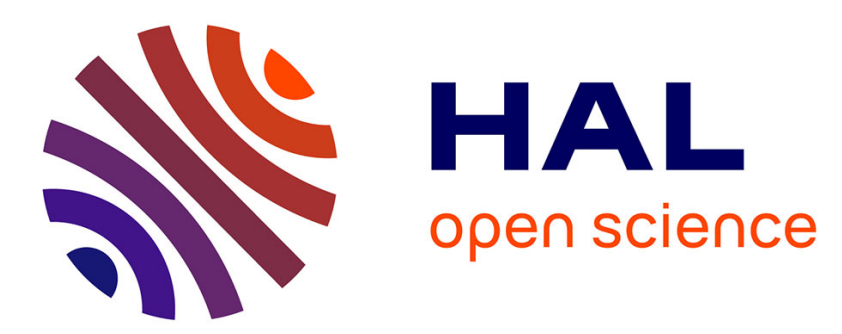

\title{
Existence de points fixes enlacés à une orbite périodique d'un homéomorphisme du plan
}

Christian Bonatti, Boris Kolev

\section{To cite this version:}

Christian Bonatti, Boris Kolev. Existence de points fixes enlacés à une orbite périodique d'un homéomorphisme du plan. Ergodic Theory and Dynamical Systems, 1992, 12 (4), pp.677-682. hal-00003268

\section{HAL Id: hal-00003268 \\ https://hal.science/hal-00003268}

Submitted on 11 Dec 2007

HAL is a multi-disciplinary open access archive for the deposit and dissemination of scientific research documents, whether they are published or not. The documents may come from teaching and research institutions in France or abroad, or from public or private research centers.
L'archive ouverte pluridisciplinaire HAL, est destinée au dépôt et à la diffusion de documents scientifiques de niveau recherche, publiés ou non, émanant des établissements d'enseignement et de recherche français ou étrangers, des laboratoires publics ou privés. 


\title{
EXISTENCE DE POINTS FIXES ENLACÉS À UNE ORBITE PÉRIODIQUE D'UN HOMÉOMORPHISME DU PLAN
}

\author{
CHRISTIAN BONATTI AND BORIS KOLEV
}

\begin{abstract}
RÉSUMÉ. Soit $f$ un homéomorphisme du plan qui préserve l'orientation et tel que $f-I d$ soit une contraction. Sous ces hypothèses, on établit l'existence, pour toute orbite périodique $\mathcal{O}$, d'un point fixe ayant un nombre d'enlacement non nul avec $\mathcal{O}$.
\end{abstract}

\section{INTRODUCTION}

Un résultat classique dû à Brouwer énonce que tout homéomorphisme du plan $\mathbb{R}^{2}$ qui préserve l'orientation et possède une orbite périodique, possède également un point fixe. Dans le même ordre d'idées, on peut montrer [4, 6] qu'un tel homéomorphisme $f$ possède un point fixe lié à cette orbite périodique, en ce sens qu'il n'existe pas de courbe de Jordan $\mathcal{C}$, bordant un disque $D$ contenant l'orbite périodique mais ne contenant pas le point fixe, et telle que $f(\mathcal{C})$ soit homotope à $\mathcal{C}$ dans le complémentaire de l'orbite périodique et du point fixe. Une question posée par John Franks dans [1] demeure toujours sans réponse :

Étant donné un homéomorphisme $f: \mathbb{R}^{2} \rightarrow \mathbb{R}^{2}$ préservant l'orientation, existe-t-il pour toute orbite périodique de $f$ un point fixe ayant un nombre d'enlacement non nul avec cette orbite périodique?

On sait que la réponse à cette question est positive pour les orbites de période 2 (voir [2]) ou de période 3 [5]. D'autre part, dans une prépublication récente, Franks [3] utilise la réponse affirmative à cette question comme étant un théorème de Handel (sans référence) : on peut donc supposer que cette question est, soit résolue, soit en passe de l'être.

Nous montrons ici qu'un raisonnement très simple et très rapide permet de répondre par l'affirmative à la question de Franks pour les orbites de toutes les périodes des homéomorphismes $f$ de $\mathbb{R}^{2}$ pour lesquels $f-I d$ vérifie un condition de Lipschitz. Plus précisément :

Théorème 1.1. Soit $f: \mathbb{R}^{2} \rightarrow \mathbb{R}^{2}$ un homéomorphisme qui préserve l'orientation et tel que $f-I d$ soit lipschitzienne de rapport $k \in[0,1]$. Alors, pour toute orbite périodique $\mathcal{O}=\left\{x, f(x), \ldots, f^{n-1}(x)\right\}$ de $f$, il existe un point fixe de $f, x_{0}$, ayant un nombre d'enlacement non nul avec $\mathcal{O}$.

Outre l'intérêt du résultat, la simplicité de la preuve met en valeur l'avantage qu'il y a à tester sur cette classe (pas trop petite) d'homéomorphismes, les conjectures concernant les homéomorphismes des surfaces.

Date: 1 Août 1991.

1991 Mathematics Subject Classification. 55M20, 54H20.

Key words and phrases. Homéomorphismes du plan, Points fixes. 


\section{Nombre D'enlacement D'un point fiXe et D'une orbite PÉRIODIQUE}

Soit $f: \mathbb{R}^{2} \rightarrow \mathbb{R}^{2}$ un homéomorphisme. On note Fix $(f)$ l'ensemble de ses points fixes. Un point $x \in \mathbb{R}^{2}$ est périodique de période $n$ si $f^{n}(x)=x$ mais $f^{k}(x) \neq x$ pour $k \in\{1,2, \ldots, n-1\}$. On note

$$
\mathcal{O}(n, f)=\left\{x, f(x), \ldots, f^{n-1}(x)\right\}
$$

l'orbite de $x$ sous $f$.

Soit $\mathrm{x}_{0} \in F i x(f), x$ un point périodique de période $n$ et $c$ un arc joignant $x$ et $f(x)$ dans $\mathbb{R}^{2} \backslash F i x(f)$, on note $\gamma_{c}$ la courbe fermée obtenue en joignant bout à bout les arcs, $c, f(c), \ldots, f^{n-1}(c)$.

On note $\omega\left(x_{0}, \gamma_{c}\right)$ le nombre d'enroulement de $\gamma_{c}$ autour de $x_{0}$ c'est à dire le nombre d'intersection algébrique d'une demi-droite générique issue de $x_{0}$ avec $\gamma_{c}$ (ce nombre est souvent appelé indice de $x_{0}$ par rapport à $\gamma_{c}$ ).

Lemme 2.1. Soient $c$ et $c^{\prime}$ deux arcs quelconques joignant $x$ et $f(x)$ dans $\mathbb{R}^{2} \backslash\left\{x_{0}\right\}$. Si f préserve l'orientation, alors $\omega\left(x_{0}, \gamma_{c}\right)-\omega\left(x_{0}, \gamma_{c^{\prime}}\right) \in n \mathbb{Z}$.

Démonstration. On a

$$
\omega\left(x_{0}, \gamma_{c}^{\prime}\right)=\omega\left(x_{0}, \gamma_{c}\right)+\sum_{k=o}^{n-1} \omega\left(x_{0}, f^{k}\left(c^{-1} c^{\prime}\right)\right) .
$$

Or si $f$ préserve l'orientation

$$
\omega\left(x_{0}, f^{k}\left(c^{-1} c^{\prime}\right)\right)=\omega\left(x_{0}, c^{-1} c^{\prime}\right),
$$

d'où $\omega\left(x_{0}, \gamma_{c}\right)-\omega\left(x_{0}, \gamma_{c^{\prime}}\right)=n \omega\left(x_{0}, c^{-1} c^{\prime}\right)$.

On peut montrer également que la valeur de $\omega\left(x_{0}, \gamma_{c}\right)(\bmod n)$ ne dépend pas du choix du point $x$ de $\mathcal{O}$ choisi pour le construire.

Définition 2.2. Avec les notations ci-dessus, on note $L k\left(x_{0}, \mathcal{O}\right)$ l'unique entier $l \in\{0,1, \ldots, n-1\}$ tel que $\omega\left(x_{0}, \gamma_{c}\right)-l \in n \mathbb{Z}$ pour un choix quelconque de $c$ et on l'appelle le nombre d'enlacement (ou linking number) du point fixe $x_{0}$ avec l'orbite périodique $\mathcal{O}$.

Remarque 2.3. L'appellation «nombre d'enlacement » est justifiée par la remarque suivante : $L k\left(x_{0}, \mathcal{O}\right)$ est aussi le nombre d'enlacement des deux orbites fermées $C_{x_{0}}$ et $C_{x}$ du champ de vecteurs canonique induit dans la suspension $T_{f}$ de $f$.

Exemple 2.4. Le point fixe de la rotation d'angle $2 k \pi / n k \in\{0, \ldots, n-$ 1\} a pour nombre d'enlacement $k$ avec l'une quelconque de ses orbites périodiques.

3. Quelques PRopriétÉS ÉLÉMENTAIRES D'Un homéomorPhisme $f$ DU PLAN, TEL QUE $f-I d$ VÉRIFIE UNE CONDITION DE LIPSCHITZ

Soit $g: \mathbb{R}^{2} \rightarrow \mathbb{R}^{2}$ une application continue. Si l'ensemble

$$
\left\{k \in \left[0, \infty\left[; \forall x, y \in \mathbb{R}^{2}:\|g(x)-g(y)\| \leq k\|x-y\|\right\}\right.\right.
$$

est non vide, on note $\operatorname{Lip}(g)$ sa borne inférieure. Sinon, on pose $\operatorname{Lip}(g)=$ $+\infty$. 
Soient $x$ et $y$ deux points de $\mathbb{R}^{2}$, on note $[x, y]$ le segment de droite joignant $x$ et $y$.

Lemme 3.1. Soit $f: \mathbb{R}^{2} \rightarrow \mathbb{R}^{2}$ un homéomorphisme tel que Lip $(f-I d) \leq 1$ et $x \in \mathbb{R}^{2} \backslash$ Fix $(f)$. Alors Fix $(f) \cap[x, f(x)]=\emptyset$.

Démonstration. Par l'absurde, supposons qu'il existe $y \in F i x(f) \cap[x, f(x)[$. On a alors :

$\|f(x)-x\|=\|(f(x)-x)-(f(y)-y)\| \leq \operatorname{Lip}(f-I d)\|x-y\|<\|f(x)-x\|$.

D'autre part $f(x) \in \mathbb{R}^{2} \backslash F i x(f)$ car $f$ est injective, ce qui conclut.

Lemme 3.2. Soit $f: \mathbb{R}^{2} \rightarrow \mathbb{R}^{2}$ un homéomorphisme tel que Lip $(f-I d) \leq$ 1 et $x \in \mathbb{R}^{2} \backslash$ Fix $(f)$. Alors, $f([x, f(x)])$ et $\left[f(x), f^{2}(x)\right]$ sont homotopes relativement à $f(x), f^{2}(x)$ dans $\mathbb{R}^{2} \backslash F i x(f)$.

Démonstration. Soit $F:[0,1]^{2} \rightarrow \mathbb{R}^{2}$ définie par :

$$
F(s, t)=(1-t)((1-s) x+s f(x))+t f((1-s) x+s f(x)) .
$$

L'image de $F$ est la réunion des segments de droite $[y, f(y)](y \in[x, f(x)])$. D'après le Lemme $3.1 y \in[x, f(x)]$ n'est pas un point fixe de $f$, donc $[y, f(y)] \subset \mathbb{R}^{2} \backslash F i x(f)$. D'où $i \mathrm{~m} F \subset \mathbb{R}^{2} \backslash F i x(f)$. D'autre part, $F(s, 0)=$ $F(0, s)=(1-s) x+s f(x)$. On obtient donc une application du disque :

$$
D=[0,1]^{2} /(s, 0) \sim(0, s)-\mathbb{R}^{2} \backslash F i x(f)
$$

telle que $F(\partial D)=\left[f(x), f^{2}(x)\right] \cup f([x, f(x)])$. On a ainsi réalisé l'homotopie.

Soient $a, b$ deux vecteurs de $\mathbb{R}^{2}$. On note $(a, b) \in[0, \pi]$ l'angle non orienté des vecteurs $a$ et $b$.

Lemme 3.3. Soit $f: \mathbb{R}^{2} \rightarrow \mathbb{R}^{2}$ un homéomorphisme tel que Lip $(f-I d) \leq 1$ et $x \in \mathbb{R}^{2} \backslash$ Fix $(f)$. Alors pour tout $y \in[x, f(x)]$ :

$$
(f(x)-x, f(y)-y)<\pi / 2 .
$$

Démonstration. On a :

$$
\|(f(x)-x)-(f(y)-y)\| \leq \operatorname{Lip}(f-I d)\|x-y\| \leq\|f(x)-x\|,
$$

et par suite $: \cos (f(x)-x, f(y)-y) \geq 0$. Si l'angle est $\pi / 2$, cela implique $y=f(y)$ ce qui est impossible en vertu du Lemme 3.1.

\section{DÉmonstration DU ThÉORÈme}

Soit $\mathcal{O}=\left\{x, f(x), \ldots, f^{n-1}(x)\right\}$ l'orbite d'un point périodique de période $n$ d'un homéomorphisme de $\mathbb{R}^{2}$ qui préserve l'orientation et tel que $\operatorname{Lip}(f-$ $I d) \leq 1$. Soit $\Gamma$ la courbe polygonale obtenue en joignant bout à bout les segments $[x, f(x)],\left[f(x), f^{2}(x)\right], \ldots,\left[f^{n-1}(x), x\right]$ (voir Figure 1 ) et soit $c=$ $[x, f(x)]$.

Lemme 4.1. Avec les notations ci-dessus, on a:

(1) $\Gamma \subset \mathbb{R}^{2} \backslash F i x(f)$,

(2) Pour tout $\mathrm{x}_{0} \in$ Fix $(f), \omega\left(x_{0}, \gamma_{c}\right)=\omega\left(x_{0}, \Gamma\right) \in\{-(n-1), \ldots, n-1\}$. 


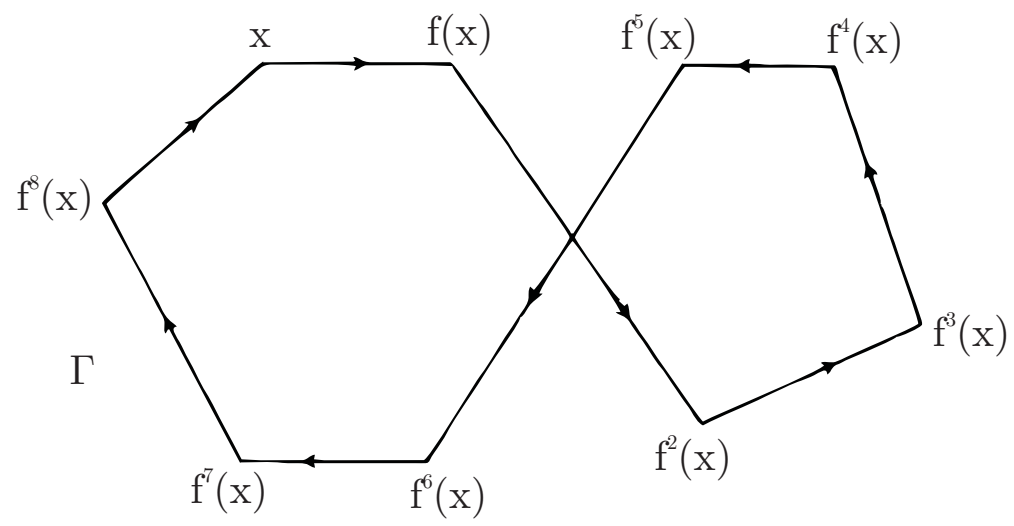

FIG. $1-$

Démonstration. L'assertion (1) résulte du Lemme 3.1. En raisonnant par récurrence et en utilisant le Lemme 3.2 , on établit que $\Gamma$ est homotope à $\gamma_{c}$ dans $\mathbb{R}^{2} \backslash F i x(f)$, d'où l'égalité $\omega\left(x_{0}, \gamma_{c}\right)=\omega\left(\mathrm{x}_{0}, \Gamma\right)$. Par ailleurs, le nombre d'enroulement de $\Gamma$ par rapport à $x_{0}$ est aussi le nombre algébrique de croisements d'une demi-droite issue de $x_{0}$ avec $\Gamma$. Ce nombre est donc nécessairement inférieur à $n-1$ en valeur absolue.

Soient $C_{1}, C_{2}, \ldots, C_{r}$ les composantes connexes bornées de $\mathbb{R}^{2} \backslash \Gamma$ (remarquer qu'il en existe au moins une, sinon $\Gamma$ serait réduit à un segment de droite ce qui est exclu en vertu du Lemme 3.3) et $C_{\infty}$ la composante connexe non bornée. Si $C \in\left\{C_{1}, C_{2} \ldots s, C_{r}\right\}$ on définit l'indice de $C$ en posant :

$$
\operatorname{Ind}(f, C)=\frac{1}{2 \pi} \int_{\partial C} d \varphi
$$

où $\varphi$ désigne une détermination continue de l'angle du vecteur $f(x)-x$ avec une direction fixe et où $\partial C$ est le bord orienté de $C$. Dans chaque composante d'indice non nul il existe au moins un point fixe de $f$. Soient $S_{0}, \ldots, S_{m-1}$ les sommets de $\partial C$ et $a_{0}, \ldots, a_{m-1}$ ses arrêtes munies de l'orientation induite par celle de $\Gamma$. En un sommet $S_{i}$, il y a deux configurations possibles quant à l'orientation des arêtes adjacentes à $S_{i}$ : ou bien ces deux arrêtes ont des orientations compatibles, ou bien il y a un changement d'orientation (voir Figure 2).

Le nombre total de changement d'orientation sur $\partial C$ est un nombre pair que l'on notera $2 p$.

Lemme 4.2. Avec les notations ci-dessus, on a $: \operatorname{Ind}(f, C)=1-p$.

Démonstration. On a :

$$
\int_{\partial C} d \varphi=\sum_{i=0}^{m-1} \int_{a_{i}} d \varphi=\sum_{i=0}^{m-1}\left(\varphi_{i}^{1}-\varphi_{i}^{0}\right),
$$

où $\varphi_{i}^{0}$ et $\varphi_{i}^{1}$ sont les valeurs respectives en $S_{i}$ et $S_{i+1}(i \in \mathbb{Z} / m \mathbb{Z})$ d'une détermination continue $\varphi_{i}$ de l'angle $f(y)-y\left(y \in a_{i}\right)$ avec la tangente 


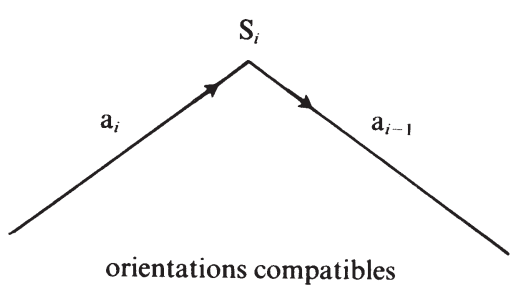

orientations compatibles

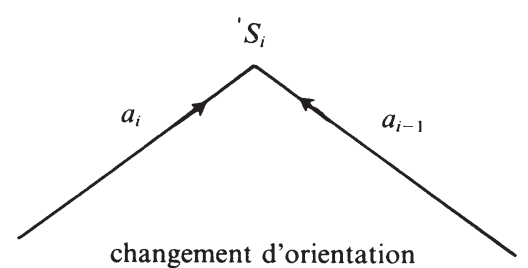

changement d'orientation

FIG. 2 -

orientée à $a_{i}$. Il résulte alors du Lemme 3.3 que $\varphi_{i}^{1}-\varphi_{i}^{0} \in[-\pi, \pi]$ (autrement dit le vecteur $f(y)-y$ ne décrit pas de tour complet lorsque $y$ parcourt $a_{i}$ ). En désignant alors par $\beta_{i} \in[0, \pi]$ l'angle intérieur à $C$ en $S_{i}$, on a (voir Figure 3) :

$\varphi_{i-1}^{1}-\varphi_{i}^{0}=\pi-\beta_{i}, \quad$ s'il n y a pas de changement d'orientation en $S_{i}$,

$\varphi_{i-1}^{1}-\varphi_{i}^{0}=-\beta_{i}, \quad$ s'il y a un changement d'orientation en $S_{i}$.

Par suite :

$$
\begin{aligned}
\operatorname{Ind}(f, C) & =\frac{1}{2 \pi}\left[\sum_{i=0}^{m-1}\left(\varphi_{i}^{1}-\varphi_{i}^{0}\right)\right] \\
& =\frac{1}{2 \pi}\left[\sum_{i=0}^{m-1}\left(\varphi_{i-1}^{1}-\varphi_{i}^{0}\right)\right] \\
& =\frac{1}{2 \pi}\left[\sum_{i=0}^{m-1}\left(\pi-\beta_{i}\right)-2 p \pi\right] \\
& =1-p .
\end{aligned}
$$

Nous pouvons maintenant établir le théorème. Le nombre d'enroulement $\omega(x, \Gamma)$ d'un point $x \in \mathbb{R}^{2} \backslash \Gamma$ ne dépendant que de la composante $C$ à laquelle il appartient, on notera $\omega(C, \Gamma)$ cette valeur commune. Il nous reste donc à établir l'existence d'une composante $C_{i}$ de $\mathbb{R}^{2} \backslash \Gamma$ telle que :

$$
\text { (1) } \operatorname{Ind}\left(f, C_{i}\right)>0 \quad \text { et } \quad(2) \quad \omega\left(C_{i}, \Gamma\right) \neq 0 .
$$

Lemme 4.3. Soit $p \in\{1, \ldots, r\}$ tel que $:\left|\omega\left(C_{p}, \Gamma\right)\right|=\sup _{i}\left|\omega\left(C_{i}, \Gamma\right)\right|$. Alors $\operatorname{Ind}\left(f, C_{i}\right)>0$.

Démonstration. Remarquons d'abord que $\omega\left(C_{\infty}, \Gamma\right)=0$ et que $\omega\left(C_{i}, \Gamma\right) \neq 0$ pour toute composante $C_{i}$ adjacente à $C_{\infty}$. Il existe donc bien $p \in\{1, \ldots, r\}$ tel que :

$$
\left|\omega\left(C_{p}, \Gamma\right)\right|=\sup _{i}\left|\omega\left(C_{i}, \Gamma\right)\right|>0 .
$$

Par l'absurde, supposons que $\operatorname{Ind}\left(f, C_{p}\right) \leq 0$. D'après le Lemme 4.2, il existe alors au moins un changement d'orientation en un des sommets $S_{k}$ de $\partial C_{p}$. 

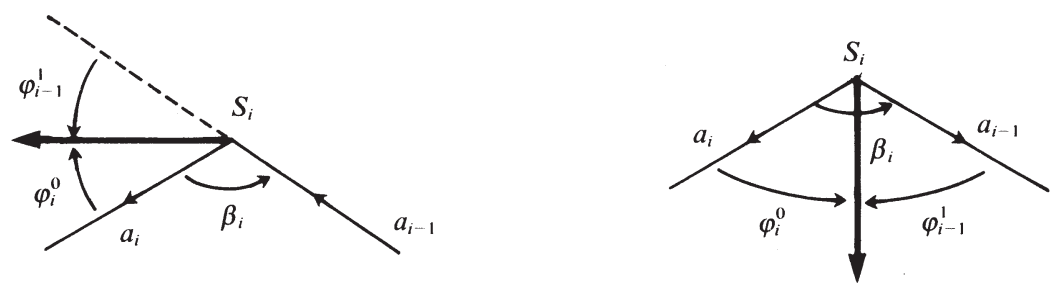

FIG. 3 -

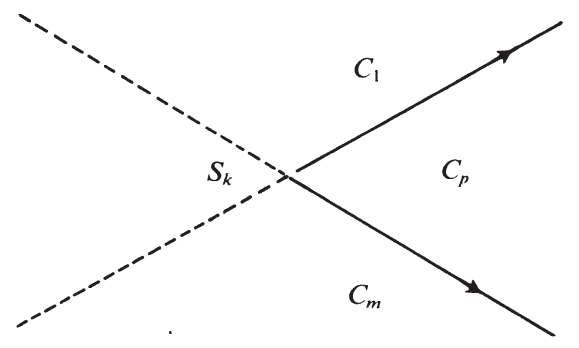

FIG. 4 -

Alors l'une des composantes $C_{l}$ adjacente à $C_{p}$ en $S_{k}$ vérifie $\omega\left(C_{l}, \Gamma\right)<$ $\omega\left(C_{p}, \Gamma\right)$ et l'autre $C_{m}$ vérifie $\omega\left(C_{m}, \Gamma\right)>\omega\left(C_{p}, \Gamma\right)$ (voir Figure 4 ). En effet, les valeurs de $\omega\left(C_{l}, \Gamma\right)$ et $\omega\left(C_{m}, \Gamma\right)$ ne dépendent que de l'orientation avec laquelle on franchit $\Gamma$ pour passer de $C_{p}$ à $C_{l}$ et $C_{m}$. Il existe donc $q \in\{l, m\}$ $(q \neq p, \infty)$ tel que :

$$
\left|\omega\left(C_{q}, \Gamma\right)\right|>\left|\omega\left(C_{p}, \Gamma\right)\right|>0
$$

ce qui contredit l'hypothèse faite sur $p$.

\section{RÉFÉRENCES}

[1] P. Boyland. Notes on dynamics of surface homeomorphisms : lectures by P. Boyland and J. Franks. notes by C. Carroll, J. Guaschi and T. Hall, August 1989, Warwick, pp. 1-48, 1989.

[2] M. Brown. Fixed points for orientation preserving homeomorphisms of the plane which interchange two points. Pacific J. Math., 143(1) :37-41, 1990.

[3] J. Franks. Geodesics on $S^{2}$ and periodic points of annulus homeomorphisms. Invent. Math., 108(2) :403-418, 1992.

[4] J.-M. Gambaudo. Periodic orbits and fixed points of a $C^{1}$ orientation-preserving embedding of $D^{2}$. Math. Proc. Cambridge Philos. Soc., 108(2) :307-310, 1990.

[5] J. Guaschi. Fixed points and linking with periodic orbits of surface diffeomorphisms and a generalization of brouwer's lemma. Preprint, Warwick Univeristy, 1990.

[6] B. Kolev. Point fixe lié à une orbite périodique d'un difféomorphisme de $R^{2}$. C. R. Acad. Sci. Paris Sér. I Math., 310(12) :831-833, 1990. 
Université de Bourgogne, Dépt. De Mathématiques, B.P. 138, 21004 Dijon Cedex, France.

E-mail address: bonatti@satie.u-bourgogne.fr

CMi, 39, rue F. Joliot-Curie, 13453 Marseille Cedex 13, France

E-mail address: kolev@cmi.univ-mrs.fr 